\title{
The mean rise velocity of pairwise-interacting bubbles in liquid
}

\author{
By L. VAN WIJNGAARDEN \\ University of Twente, Enschede, The Netherlands
}

(Received 20 July 1988 and in revised form 16 November 1992)

This paper is concerned with calculations regarding a collection of small gas bubbles rising under buoyancy in a clear liquid. For dilute mixtures interactions can be restricted to those between two bubbles. In the analysis of binary interactions it is assumed that the Reynolds number for relative motion between bubbles and liquid is large, that surfactants, if present, do not modify the condition of zero tangential stress at the bubble-liquid interface, and that bubbles bounce at an encounter.

A two-bubble probability density is derived from the analysis, valid on a short timescale associated with the interaction. It is shown that on a long timescale, based on viscous dissipation, clustering together of pairs takes place, most likely even when triple encounters are allowed for. An analysis is given of the vertical motion of pairs, followed by a calculation of the mean vertical bubble velocity with help of the (short timescale) probability density function. The result is compared with experimental data.

\section{Introduction}

Anyone who observes collections of small air bubbles rising in water will notice the erratic motion of individual bubbles. This is due in part to shape oscillations. For isolated bubbles rising under buoyancy this happens when the size exceeds a critical value. With a suspension of bubbles the erratic motion also occurs under conditions in which a bubble, when isolated, would follow a purely vertical path. The deviations of the trajectory from a straight line are caused by hydrodynamic interactions between the bubble considered and other ones.

The motion of other bubbles generates a flow field which exerts a pressure force and a viscous force on the test bubble. These forces have a stochastic character and result in stochastic motion of individual bubbles. Further they lead to deformation of the test bubble. Both the forces and the deformation depend in such a complicated way on the flow field that it is very hard, if not impossible, to describe these in terms of flow parameters.

We shall start with assuming that the bubbles are small enough for surface tension to keep them spherical. After having obtained results for spherical bubbles, we shall discuss the influence of deformations. Further we shall assume that surfactants occur in low enough concentration to permit the condition of zero tangential shear stress on the bubbles. When in addition the diameter of the bubbles is of the order of $1 \mathrm{~mm}$, the Reynolds number for the relative motion is of the order $10^{2}$ and the flow field can with sufficient accuracy be described by potential flow theory.

These assumptions have been made previously in work on bubbly flow, e.g. in Biesheuvel \& van Wijngaarden (1984). In that paper average hydrodynamic equations were derived in such very dilute suspensions that the average velocity of rise is equal 
to the speed of rise of an isolated bubble. Further, it is well known that for low concentration binary interactions dominate, and that this leads to a correction term of the form $\lambda \alpha$, where $\lambda$ is a constant and $\alpha$ is the volume fraction of the bubbles. Hence, at low concentration, and denoting by $U_{\infty}$ the velocity of rise of an isolated bubble, one expects the average velocity of rise of a dilute suspension to behave as

$$
U_{\infty}(1-\lambda \alpha) \text {. }
$$

However, experiments by Kapteyn (1989), and also van Wijngaarden \& Kapteyn (1990), show for $0.02<\alpha<0.15$ a behaviour

$$
V(1-\lambda \alpha),
$$

where the constant $V$ is less than $U_{\infty}^{\prime}$. In the interval $0<\alpha<0.02$, the average velocity of rise seems to decrease more rapidly than linearly with $\alpha$, a behaviour which awaits explanation. Anyway, both the experiments by Kapteyn and by others, such as Bouré (1988), show for low $\alpha$, in our case $0.02<\alpha<0.15$, a linear term in the average velocity of rising.

In order to calculate this average velocity, or other average quantities, it suffices at low values of $\alpha$ to know the two-particle probability distribution function (Batchelor 1974). For this the dynamics of two-particle interactions have to be investigated. This has been done in work by Biesheuvel \& van Wijngaarden (1982), Biesheuvel (1984) and recently in Kok (1989). Some results of the work by Kok (1989) will be discussed in $\$ 2$. They will enable us in $\S 6$ to find an approximate expression for the pair probability distribution function. This will be used to calculate the average velocity $\langle u\rangle$ of a bubbly suspension for low concentration $\alpha$. The result will be compared with experiments.

Hence, three stages can be distinguished in this paper. In the first the dynamics of interactions in pairs is considered. In the second an equilibrium probability distribution function is derived. In the third stage the dynamics of vertical motion is dealt with.

\section{The relative motion in a pair of bubbles}

Imagine a large cylindrical vessel with radius $\bar{R}$, in which a large number of bubbles rise under buoyancy. We assume that all bubbles have the same size (we disregard the growth in size in the vertical direction due to decreasing hydrostatic head). The volume concentration of the bubbles $(\alpha)$ is uniform. Owing to the erratic paths of the bubbles any transport quantity can be known only as a mean, or average quantity. For the calculation of the average velocity of rising we may consider the velocity of a test bubble in the presence of all others. For low $\alpha$ the configuration of all other bubbles can be reduced to just one other bubble in order to obtain results correct to order $\alpha$ (Batchelor 1972, 1974). The reason for this is that the probability of finding another particle in a sphere around the test particle with radius comparable with the bubble radius is of order $\alpha$, the probability to find two bubbles there of order $\alpha^{2}$, etc. One may therefore restrict to interactions between two bubbles.

Trajectories followed by pairs in their phase space were calculated by Biesheuvel (1984) for inviscid fluid. Even without viscous forces, this can only be done numerically. Numerical calculations that include viscous forces were made by Kok (1989). Since we shall use some of his results we shall briefly describe his work here. The envisaged configuration is sketched in figure 1.

Two spherical bubbles with radius $a$, immersed in liquid, have their line of centres at an angle $\theta$ with the vertical direction. At time $t=0$ they are released with an initial angle $\theta_{0}$. The distance between the bubbles is denoted by $2 R$. We assume that the 


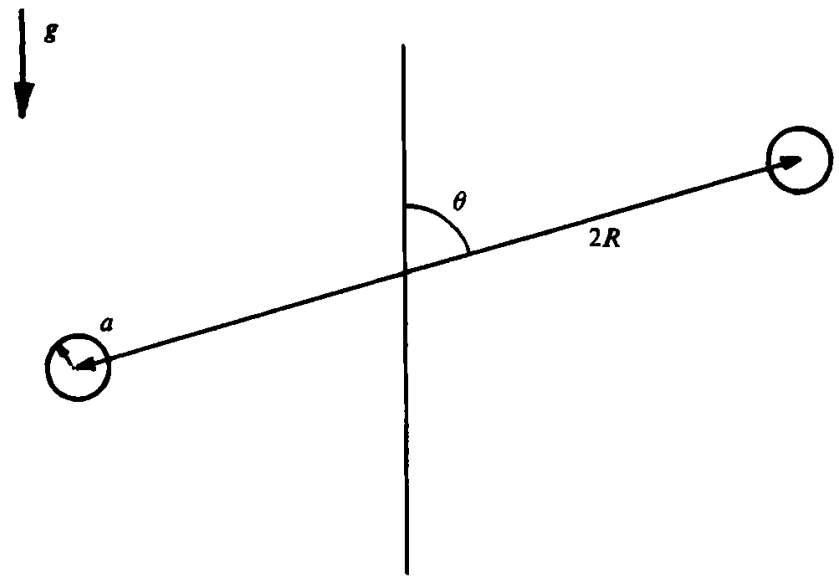

FIGURE 1. A bubble pair rising in water under buoyancy. They have radius $a$, are separated by a distance $2 R$, while their line of centres is at an angle $\theta$ with the direction of gravity, indicated $g$.

influence of surfactive agents on the boundary condition at the interface between gas and liquid can be neglected. Then, in view of the negligibly small viscosity of the gas inside the bubbles, the tangential stress at the interface must vanish. The viscous force on a rising bubble, under the further assumption of a Reynolds number large enough to make inertia forces dominate over viscous forces, was calculated by Levich (1962) and by Moore $(1963,1965)$ from the dissipation in the liquid. For vanishingly small values of $a / R$ the equilibrium velocity of rising follows from the viscous force, given by Levich as

$$
12 \pi \mu a U_{\infty},
$$

where $\mu$ is the dynamic viscosity of the liquid. The buoyancy force is

$$
\frac{4}{3} \pi a^{3} \rho g \text {, }
$$

where $\rho$ is the density of the fluid and $g$ the acceleration due to gravity. Introducing the kinematic viscosity of the liquid $(v)$, it follows that

$$
U_{\infty}=g a^{2} / 9 \nu \text {. }
$$

It follows that the Reynolds number is $g a^{3} / 9 v^{2}$. For water and air bubbles of $1 \mathrm{~mm}$ radius this is $10^{2}$ approximately, which is large enough for Levich's result to be valid. With two bubbles in interaction the vertical velocity, $u$ say, of the centre of mass fluctuates about a value which is close to $U_{\infty}$.

Under these conditions the velocities in the fluid around the bubbles can be derived from a flow potential. This was given by, among others, van Wijngaarden (1976) as an infinite series of spherical harmonics in terms of $\theta$ and $a / R$ and centred in one of the bubbles in a pair. This allows calculation of the kinetic energy in the liquid. By an extension of the work of Moore $(1963,1965)$ it is possible to derive the viscous forces on each of the bubbles from the calculated dissipation in the liquid. This, in contrast to the case of the flow past solid bodies, takes place in the whole of the liquid and not mainly in the boundary layer. Starting from expressions for the kinetic energy and for the dissipation, both derived from the velocity potential, Kok (1989) used Lagrange's principle to derive equations of motion, both for the relative motion as well as for the centre of mass. Subsequently he solved these equations numerically for various values of the initial angle $\theta_{0}$ and the initial rising velocity $u_{0}$.

In figure 2 some paths are shown of one bubble with respect to another located at 

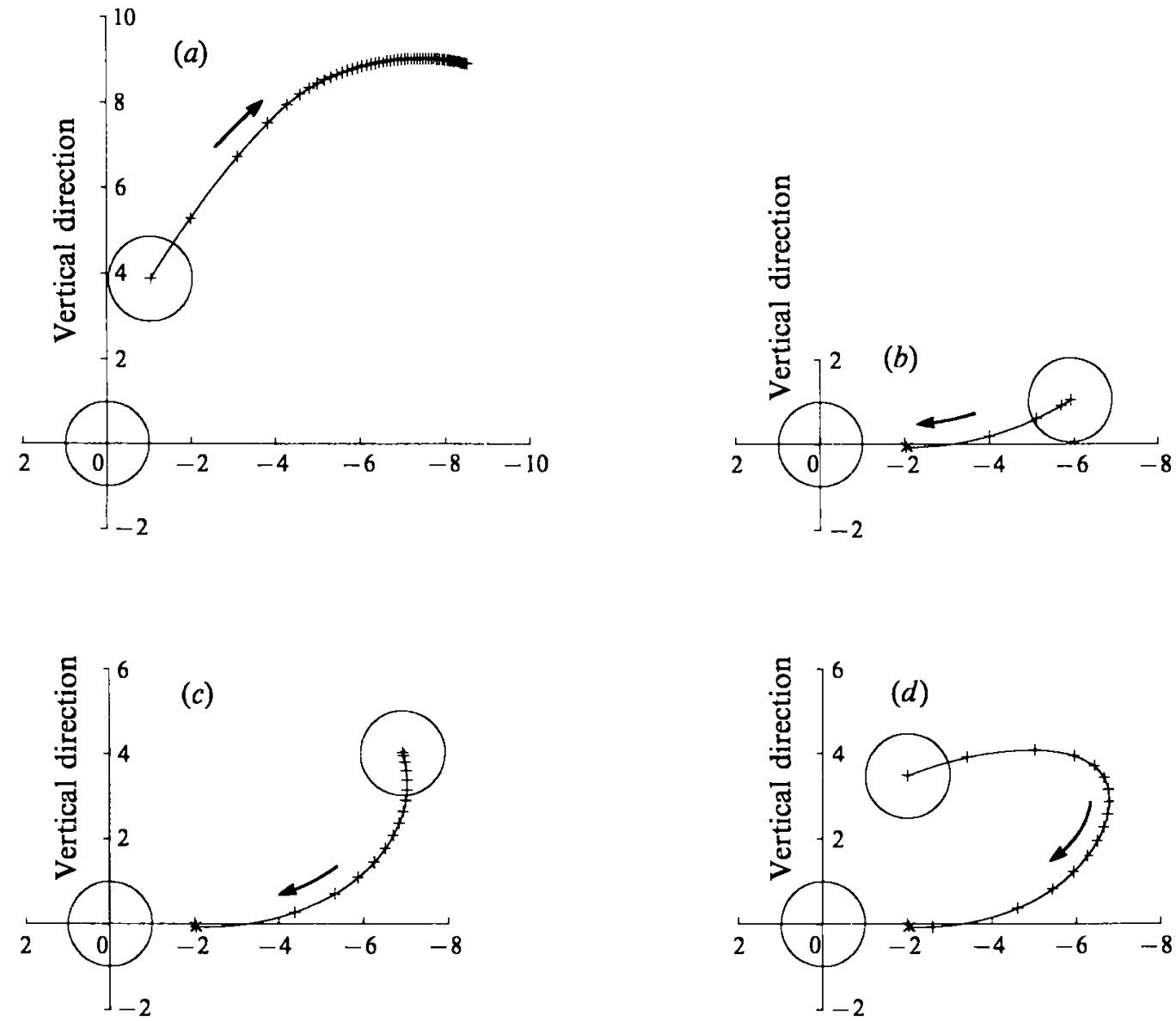

Figure 2. Numerically calculated trajectories of one bubble in a pair with respect to the other, in relative motion following their release in stagnant water. The initial separation is $2 R_{0}$, the initial angle of the line of centres with the vertical direction is $\theta_{0}$. In the expansion in terms of $(a / R) 50$ terms are taken into account. The Reynolds number is 200 in all pictures. The unit distance along the axes corresponds with the bubble radius. The arrows indicate the direction of movement along the trajectories. (a) $\theta_{0}=15^{\circ}, 2 R_{0}=4 a ;(b) \theta_{0}=80^{\circ}, 2 R_{0}=6 a ;(c) \theta_{0}=60^{\circ}, 2 R_{0}=8 a ;(d) \theta_{0}=30^{\circ}$, $2 R_{0}=4 a$.

the origin in the figure, for $u_{0}=U_{\infty}$. From these examples and from computations of other trajectories we conclude that there are two types of trajectory: (i) trajectories as in figure 2(a) such that separation always grows, albeit eventually at a vanishingly small rate; (ii) trajectories as in figure $2(b), 2(c)$ and $2(d)$, such that two bubbles come together along the line $\theta=\frac{1}{2} \pi$, sometimes after an initial separation. In these calculations terms up to and including $(a / R)^{50}$ are taken into account. At the present author's request Dr Kok was kind enough to repeat the numerical calculations, this time leaving out terms in the interaction force of order $(a / R)^{5}$ and higher. The difference between the result of these calculations and the previous ones that included terms of order $(a / R)^{50}$ is so small that it can hardly be shown in a figure. Taking into account these extremely small differences we adopt in the following the approximation of leaving out terms in the force balance of order $(a / R)^{5}$ and higher. This allows a partly analytical treatment. Terms in the expression for the potential can be associated with multipoles. The present approximation amounts to taking into account the dipoles and neglecting multipoles of higher order. 
The equation for the relative motion of two bubbles describes the balance between inertia forces (effect of virtual mass), attractive/repulsive forces (due to pressure terms other than the $\partial / \partial t$ term in Bernoulli's equation) and viscous forces. The ratio between the inertia forces and viscous forces contains a relaxation time. Leaving aside deviations from spherical shape, the virtual mass of an isolated bubble is

$$
m=\frac{2}{3} \pi \rho a^{3} .
$$

The frictional force on an isolated bubble with velocity $U_{\infty}$ is given by (2.1). The relaxation time is therefore

$$
\tau=\frac{\frac{2}{3} \pi \rho a^{3}}{12 \pi \mu a}=\frac{a^{2}}{18 \nu} .
$$

For a bubble with $a=1.5 \times 10^{-3} \mathrm{~m}$ rising in water, $\tau=0.125 \mathrm{~s}$. For a bubble with $a=$ $10^{-3} \mathrm{~m}$ the corresponding value for $\tau$ is $6 \times 10^{-2} \mathrm{~s}$. We can, as these examples show, consider $\tau$ to be typically of order $10^{-1} \mathrm{~s}$. The strength of the dipole depends on the vertical velocity of the centre of mass of the pair, which we denote by $u$, as well as on the velocity of this centre in a horizontal plane. The latter is, in a rising mixture, negligibly small in comparison with the vertical velocity. Without viscous terms the equations for the relative motion, describing the change of $R$ and of $\theta$, were given in Biesheuvel \& van Wijngaarden (1982). Complemented with viscous terms they are, as given in Kok (1989),

$$
\begin{gathered}
\frac{\mathrm{d}^{2} R}{\mathrm{~d} t^{2}}-R\left(\frac{\mathrm{d} \theta}{\mathrm{d} t}\right)^{2}-\frac{9}{16} \frac{a^{3}}{R^{4}}\left(\frac{\mathrm{d} R}{\mathrm{~d} t}\right)^{2}+\tau^{-1} \frac{\mathrm{d} R}{\mathrm{~d} t}=\frac{9}{32} \frac{a^{3} u^{2}}{R^{4}}\left(3 \cos ^{2} \theta-1\right), \\
R \frac{\mathrm{d}^{2} \theta}{\mathrm{d} t^{2}}+2 \frac{\mathrm{d} R}{\mathrm{~d} t} \frac{\mathrm{d} \theta}{\mathrm{d} t}+\tau^{-1} R \frac{\mathrm{d} \theta}{\mathrm{d} t}=\frac{9}{32} \frac{a^{3} u^{2}}{R^{4}} \sin 2 \theta .
\end{gathered}
$$

The velocity $u$ in the vertical direction is close to $U_{\infty}$; the difference is of order $U_{\infty} a^{3} / R^{3}$. This difference leads, when inserted in the right-hand side of (2.5) and of (2.6), to terms of order $(a / R)^{6}$, which are negligibly small in the present approximation. We may replace $u$ by $U_{\infty}$, therefore, in the right-hand sides of (2.5) and (2.6). The interaction between the bubbles has a timescale $a / U_{\infty}$. The ratio between this and the viscous relaxation time $\tau$ appears naturally when we make (2.5) and (2.6) dimensionless with the relations

$$
R=a \eta, \quad t=T a / U_{\infty} .
$$

In terms of $\eta$ and $T,(2.5)$ and (2.6) become

$$
\begin{gathered}
\frac{\mathrm{d}^{2} \eta}{\mathrm{d} T^{2}}-\eta\left(\frac{\mathrm{d} \theta}{\mathrm{d} T}\right)^{2}-\frac{9}{16} \frac{1}{\eta^{4}}\left(\frac{\mathrm{d} \eta}{\mathrm{d} T}\right)^{2}+\sigma^{-1} \frac{\mathrm{d} \eta}{\mathrm{d} T}=\frac{9}{32} \frac{3 \cos ^{2} \theta-1}{\eta^{4}}, \\
\eta \frac{\mathrm{d}^{2} \theta}{\mathrm{d} T^{2}}+2\left(\frac{\partial \eta}{\mathrm{d} T}\right)\left(\frac{\mathrm{d} \theta}{\mathrm{d} T}\right)+\sigma^{-1} \eta \frac{\mathrm{d} \theta}{\mathrm{d} T}=\frac{9}{32} \frac{\sin 2 \theta}{\eta^{4}} .
\end{gathered}
$$

The new parameter $\sigma$ in these dimensionless equations is the ratio of the relaxation time $\tau$ and the interaction time $a / U_{\infty}$ :

$$
\sigma=U_{\infty} \tau / a .
$$

(Note that, apart from a numerical factor, $\sigma$ is the Reynolds number.) It is in general a quantity of order $10^{2}$. A typical velocity of rise is $U_{\infty}=0.3 \mathrm{~m} / \mathrm{s}$. Taking $a \sim 10^{-3} \mathrm{~m}$ and $\tau \sim 10^{-1} \mathrm{~m}$, we have $\sigma=30$. 
Since $\sigma^{-1}$ is a small quantity, one could start with neglecting the viscous terms and take $\sigma^{-1}=0$. This was done in Biesheuvel \& van Wijngaarden (1982). In that case all trajectories lead eventually to contact. In the range $0<\theta<\frac{1}{2} \pi$, the rate of change $\mathrm{d} \theta / \mathrm{d} T$ is positive, with $\sigma^{-1}=0$, as follows from (2.9). For $\pi>\theta>\frac{1}{2} \pi$ the line of centres is rotated back to $\theta=\frac{1}{2} \pi$. Hence, the value $\theta=\frac{1}{2} \pi$ is a stable equilibrium position. This follows also from linear stability analysis of (2.8) and (2.9). The central component of the interaction force is attractive when $\theta>\cos ^{-1} \sqrt{ } 3$ and therefore the two bubbles in a pair end up by approaching each other along a line inclined to gravity at an angle in the vicinity of $\theta=\frac{1}{2} \pi$.

The action of viscosity does not essentially change this. The only difference is that for angles $\theta$ in the range $0<\theta<\cos ^{-1} \sqrt{ } 3$ the separation grows very slowly. Analysis of (2.8) shows that for $t \gg \tau$,

$$
\eta \sim \sigma^{\frac{2}{5}}(t / \tau)^{\frac{1}{5}} .
$$

The growth rate is therefore small. (In the numerical experiments the timescale is not long enough and the motion, as in figure 2(a), apparently ceases.) Nevertheless, for these starting positions also, the angle $\theta$ ends up in the vicinity of $\theta=\frac{1}{2} \pi$. This means that the value $\theta=\frac{1}{2} \pi$ has a much higher probability than other values of $\theta$. In the following we shall disregard positions with the line of centres at angles other than $\frac{1}{2} \pi$. This amounts to taking all the lines of centres in a horizontal plane. This certainly affects the results quantitatively. The numerical simulations show that when a bubble comes within the region of influence of another, their line of centres turns into a horizontal position in a time equal to a multiple of $a / U_{x}$. During this period the line of centres is not horizontal and such positions are neglected here. Qualitatively this will not affect the outcome much because, once the line of centres is horizontal, it remains so. This means that within the lifetime of a pair the time in which the line of centres is not in a horizontal plane forms a negligible fraction.

We have seen that eventually all binary interactions lead to contact. This brings us to the important question of what happens when two bubbles meet.

\section{Phenomena at the close encounter of two bubbles}

Together with the numerical simulation, Kok (1989) carried out experiments with pairs of bubbles to verify the theory. He found that the actual trajectories are fairly well predicted by the theory which we have discussed in $\$ 2$. In this section we discuss the interesting phenomena which Kok found regarding close encounters.

Figure 3 shows two pictures, one concerning the encounter of two bubbles in hyperfiltrated water, the other in singly filtrated water. It appears that in the latter case bubbles bounce at encounter, whereas in hyperfiltrated water the two bubbles coalesce. Apparently the difference in behaviour has to do with surfactive agents. These influence both the occurrence of coalescence and the boundary condition prevailing at the interface. The two asymptotic cases are: at zero contamination there is coalescence and a zero tangential stress condition applies at the interface. At sufficiently high contamination a no-slip boundary condition holds, since the surfactives immobilize the interface, and also the bubbles bounce. The behaviour in between these extremes is neither well understood nor well documented; for a recent review, see Chesters (1991). The experience of both Kok (1989) and van Wijngaarden \& Kapteyn (1990) is that in singly filtrated water the friction force is given with good accuracy by (2.1) for bubbles of radius of about $1 \mathrm{~mm}$ whereas even at very small amounts of contamination bubbles bounce at an encounter. Kirkpatrick \& Lockett (1974) find that in a dilute cloud even in pure water coalescence is virtually absent. 


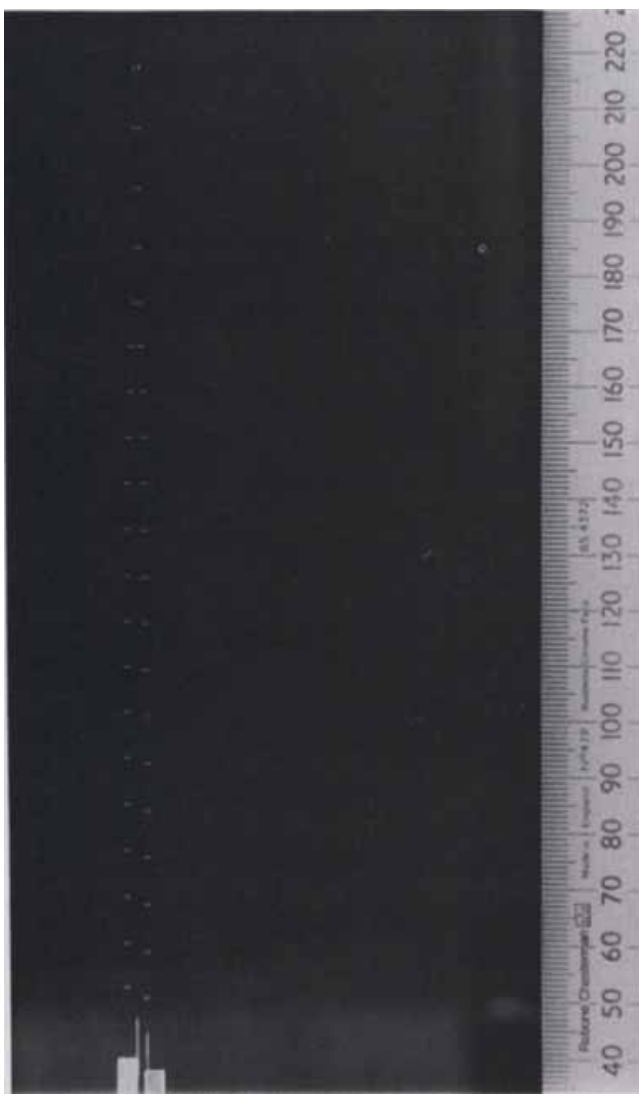

(a)

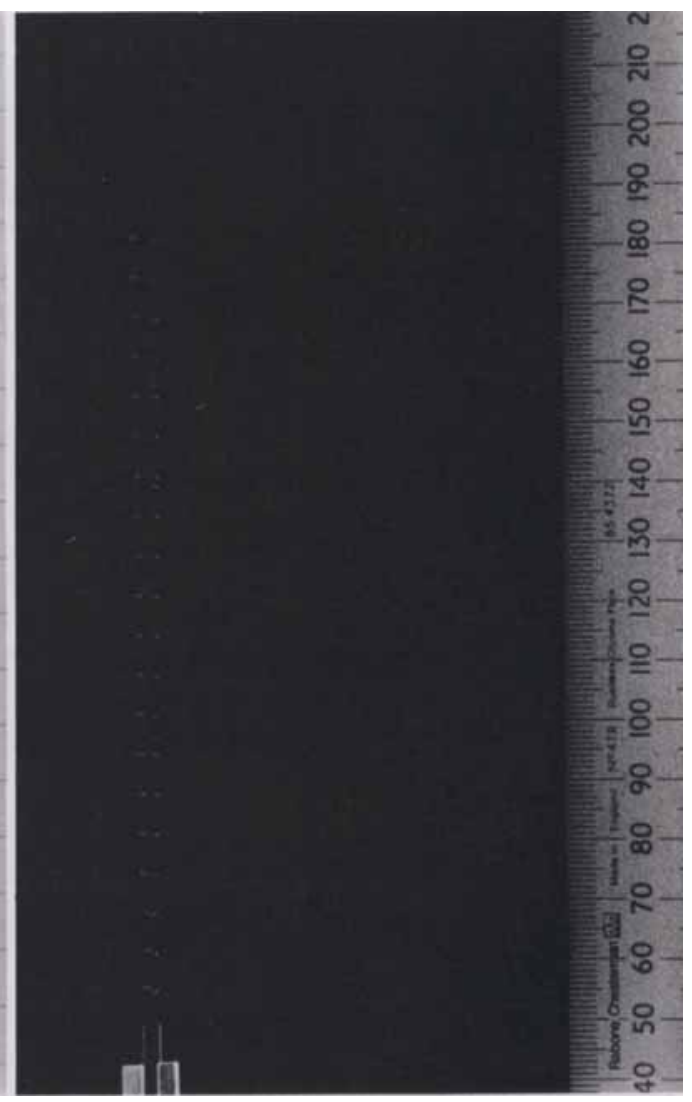

(b)

Figure 3. The interaction of two air bubbles rising in water. In (a) the water is hyperfiltrated. The two bubbles approach each other along a horizontal path and eventually coalesce. In $(b)$ the water is filtrated once. The bubbles have just bounced and then move apart, again along a horizontal path. From Kok (1989).

Based on these experimental findings, we shall assume that bubbles in a pair bounce at encounter. This encounter is, as we recall, predicted by the numerical simulation to be along a line $\theta=\frac{1}{2} \pi$. In figures $3(a)$ and $3(b)$ this orientation is observed in the experiments also. We restrict the analysis therefore to encounters along that direction. The question may be asked whether after rebound the relative velocity of the two bubbles is in the direction in which they approached each other. For purely spherical bubbles this obviously is the case, by symmetry.

In reality bubbles deform, unless they are extremely small. In figure 4(a) two bubbles are sketched approaching each other along a line perpendicular to the direction of gravity. The velocity of the centre of mass of the pair has a vertical component only. Owing to the attractive force the bubbles have in addition equal but opposite velocities along their line of centres. Owing to the non-uniform pressure field the bubbles are deformed. The shape is determined by a balance between surface tension and hydrodynamically induced pressure forces along the surface. It appears that, because the bubbles cannot support a net force, the shape is approximately an oblate spheroid with principal short axis along the resultant velocity of the deformed sphere. When the bubbles are still far apart this principal axis is vertical, but during approach it turns as indicated in figure $4(a)$. 


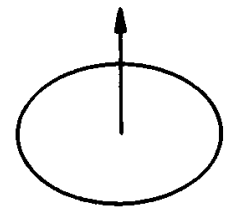

(1)

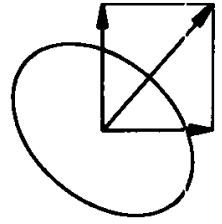

(2)

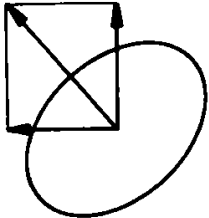

(2)

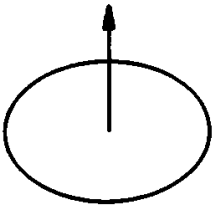

(1)

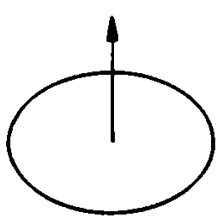

(4)

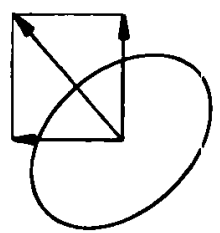

(3)

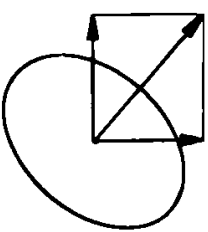

(3)

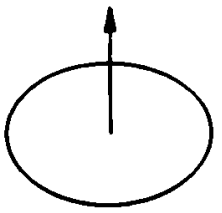

(4)

Figure 4. An encounter between two rising bubbles. The arrows indicate the velocity with respect to the liquid. (a) In positions (1) the bubbles are far apart, their relative velocity is negligible. In positions (2) they 'turn' towards each other, as explained in the text. (b) The two bubbles in a pair move away from each other (3). In positions (4) the original shape is recovered.

There is only a buoyancy force on the pair in the vertical direction. After the rebound the centre of mass of the pair has a velocity therefore only in the vertical direction and the motion will look like that sketched in figure 4(b). Again, at a large distance the initial shape will be recovered.

The picture given here of a binary encounter needs modification perhaps, when the reflective symmetry in figure 4 is absent. This is the case when the instantaneous shape on approach is not entirely due to buoyancy on the bubble itself and the motion induced by the other bubble in a pair. Additional induced velocities are due to other bubbles. In the present picture in which only pair interactions are allowed for, it seems reasonable to assume that an encounter along $\theta=\frac{1}{2} \pi$ results after the rebound in a relative motion also in that direction. Further we shall assume a spherical shape first and later on discuss the effect of deformations, in particular into oblate spheroids as in figure 4. The influence of the deformation into spheroidal form on added mass and viscous resistance is dealt with in van Wijngaarden (1991).

\section{Analysis of relative motion}

With $\mathrm{d} \theta / \mathrm{d} T=0$ and $\theta=\frac{1}{2} \pi,(2.9)$ is satisfied and (2.8) can be integrated to give

$$
2\left(\frac{\mathrm{d} \eta}{\mathrm{d} T}\right)^{2}=\left[1-\exp \left\{\frac{3}{8}\left(\eta_{\mathrm{m}}^{-3}-\eta^{-3}\right)\right\}\right]
$$


In this relation $\eta_{\mathrm{m}}=R_{\mathrm{m}} / a$ is an integration constant. At the maximum dimensionless separation of $2 \eta_{m}$ the relative velocity is zero. A simplification which facilitates the following analysis is to approximate the right-hand side of (4.1) by

$$
\left(\frac{\mathrm{d} \eta}{\mathrm{d} T}\right)^{2}=\frac{3}{16}\left(\eta^{-3}-\eta_{\mathrm{m}}^{-3}\right)
$$

or in physical variables

$$
\left(\frac{\mathrm{d} R}{\mathrm{~d} t}\right)^{2}=\frac{3}{16} \frac{a^{3} U_{\infty}^{2}}{R_{\mathrm{m}}^{3}}\left\{\left(\frac{R_{\mathrm{m}}}{R}\right)^{3}-1\right\} .
$$

The relative error in $\mathrm{d} \eta / \mathrm{d} T$ is $\frac{3}{32}\left(\eta^{-3}-\eta_{\mathrm{m}}^{-3}\right)$. With $\eta_{\mathrm{m}}=4$ this is zero at $\eta / \eta_{\mathrm{m}}=1$ and at most $9 \%$ at $\eta=1$. We shall proceed therefore with (4.3) and (4.2) (when physical variables are required). In terms of the governing differential equations, the approximation of (4.1) by (4.2) is equivalent with the neglect of the term with $(\mathrm{d} \eta / \mathrm{d} T)^{2}$ in (2.8).

From (4.2) it follows that the time spent between $\eta=\eta_{\mathrm{m}}$ and $\eta=1$ is a numerical constant times $\eta_{\mathrm{m}}^{\frac{5}{2}}\left(1-\eta_{\mathrm{m}}^{-3}\right)^{\frac{1}{2}}$. For $\eta_{\mathrm{m}}=2$, which means a maximum separation of four bubble radii, this is 5.3. Remembering that the timescale is $a / U_{\infty}$ and that $\sigma$, as defined in (2.10), is of order $10^{2}$, we conclude that in general a considerable number of oscillations can be performed before the relative motion in a pair is exhausted by viscous friction.

We now analyse the influence of viscosity on the motion in a pair, from $\eta=\eta_{\mathrm{m}}$ to $\eta=1$. With the neglect of $(\mathrm{d} \eta / \mathrm{d} T)^{2}(2.8)$ becomes

$$
\frac{\mathrm{d} \eta}{\mathrm{d} T}\left\{\sigma^{-1}+\frac{\mathrm{d}}{\mathrm{d} \eta}\left(\frac{\mathrm{d} \eta}{\mathrm{d} T}\right)\right\}+\frac{9}{32} \eta^{-4}=0 .
$$

We introduce

$$
\tilde{\eta}=\sigma^{\frac{2}{5}}=\tilde{R} / a .
$$

Analysis of (4.4) allows one to distinguish between three intervals.

(i) $0<1-\eta / \eta_{\mathrm{m}}<0.14\left(\tilde{\eta} / \eta_{\mathrm{m}}\right)^{5}$.

Here the relative velocity along the line of centres is still small (though $\mathrm{d}^{2} \eta / \mathrm{d} T^{2}$ is not) and there is a balance between the inertia force and the attractive force,

$$
\frac{\mathrm{d} \eta}{\mathrm{d} T} \frac{\mathrm{d}}{\mathrm{d} \eta}\left(\frac{\mathrm{d} \eta}{\mathrm{d} T}\right)+\frac{9}{32} \eta^{-4}=0 .
$$

(ii) $0.14\left(\tilde{\eta} / \eta_{\mathrm{m}}\right)^{5}<1-\eta / \eta_{\mathrm{m}}<1-\tilde{\eta} / \eta_{\mathrm{m}}$.

In this region the acceleration force is small and there is a balance between friction and attraction,

$$
\sigma^{-1} \frac{d \eta}{d T}+\frac{9}{32} \eta^{-4}=0
$$

(iii) $1<\eta<\tilde{\eta}$.

Here there is, as in region (i), a balance between inertia and attraction, since

$$
\frac{\mathrm{d}}{\mathrm{d} \eta}\left(\frac{\mathrm{d} \eta}{\mathrm{d} T}\right) \gg \sigma^{-1} .
$$

The relative motion is governed, as in zone (i), by (4.6).

Here it is assumed that $\tilde{\eta}<\eta_{\mathrm{m}}$. As an example, the three regions discussed here are summarized in figure 5 for the case $\eta_{\mathrm{m}}=10$ and $a=1.5 \times 10^{-3} \mathrm{~m}$. In the figure physical, rather than dimensionless, quantities are used. Near $R=a$ the acceleration 


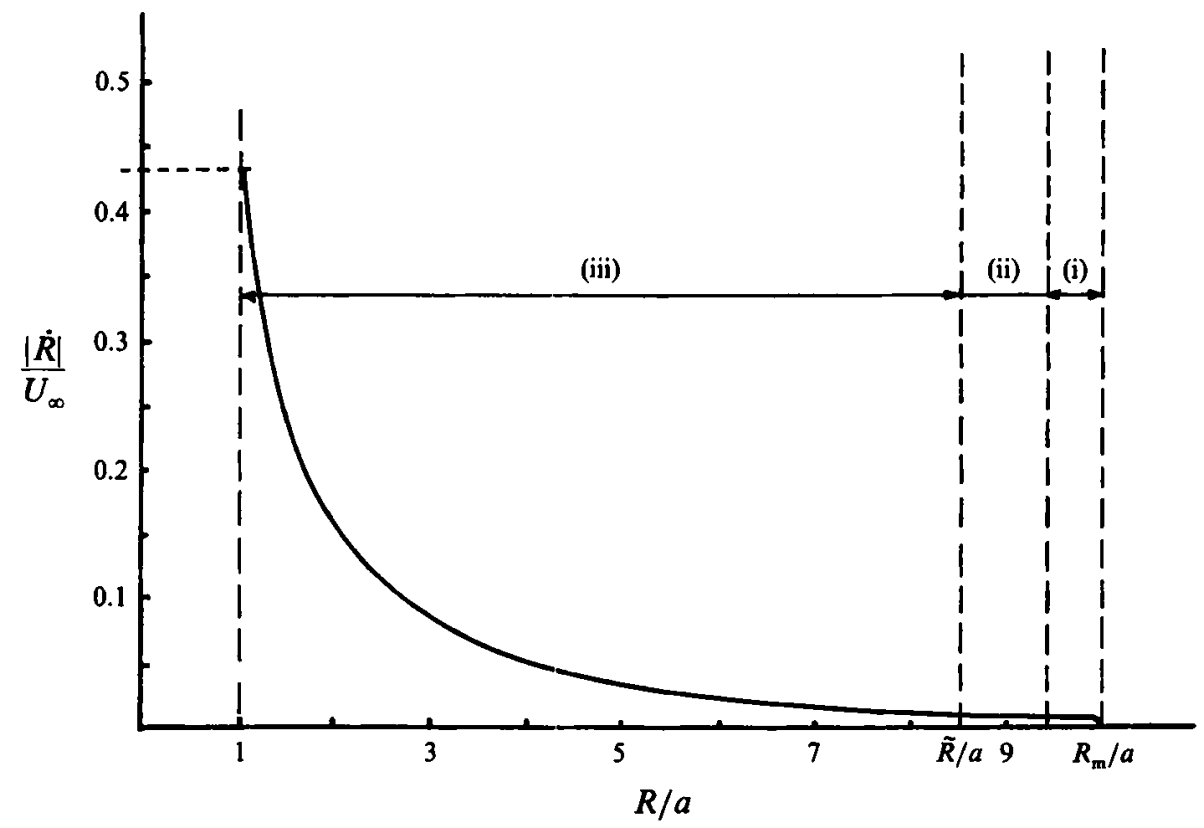

FIGURE 5. The relative velocity in a bubble pair as a function of $R / a$ for $R_{\mathrm{m}} / a=10$. In the regions indicated (i) and (iii) the relative motion is dominated by inertia. Only in (ii) is viscous friction important.

is very rapid and the bubbles approach each other with a velocity $0.43 U_{\infty}$. After rebound the same regions, as discussed above, are passed through but now in the opposite order.

From this analysis we draw the conclusion that, provided $\sigma$ is large, the relative motion can be described by (4.6), or the integrated form (4.2) of this equation. We repeat that for $\sigma \gg 1$ the two bubbles in a pair perform many oscillations before friction exhausts the motion. Of course, on a timescale longer than 5 or 6 , say, oscillations, we must be aware that $R_{\mathrm{m}}$ is not a constant but a parameter which decreases through the action of viscosity. The way $R_{\mathrm{m}}$ decreases and the consequences which this has on the behaviour of a bubbly suspension form the subject of the following section.

\section{The effect of viscous friction}

Multiplication of (4.4) with $\mathrm{d} \eta / \mathrm{d} T$ allows this equation to be written as

$$
\frac{\mathrm{d}}{\mathrm{d} T}\left\{\frac{1}{2}\left(\frac{\mathrm{d} \eta}{\mathrm{d} T}\right)^{2}-\frac{3}{32} \eta^{-3}\right\}=-\sigma^{-1}\left(\frac{\mathrm{d} \eta}{\mathrm{d} T}\right)^{2}
$$

Since $\sigma$ is large, it follows from (5.1) that on the timescale $T$ the quantity

$$
E=\frac{1}{2}\left(\frac{\mathrm{d} \eta}{\mathrm{d} T}\right)^{2}-\frac{3}{32} \eta^{-3}
$$

is a constant, equal to (cf. (4.2))

$$
E=-\frac{3}{32} \eta_{\mathrm{m}}^{-3} \text {. }
$$

This admits the following interpretation. The force $-\frac{9}{32} \eta^{-4}$ in (2.8) can be derived from a potential $-\frac{3}{32} \eta^{-3}$. Without friction the sum of kinetic energy and potential energy, $E$, 
is a constant, equal to $-\frac{3}{32} \eta_{\mathrm{m}}^{-3}$. Owing to viscosity $E$ decreases slowly. The timescale for this is $\tau$. Introducing $\tilde{t}=t / \tau$, we have, from (2.7), (2.10) and (5.1)

$$
\frac{\mathrm{d}}{\mathrm{d} \tilde{t}} E=-\left(\frac{\mathrm{d} \eta}{\mathrm{d} T}\right)^{2} .
$$

Since, for large $\sigma, \mathrm{d} E / \mathrm{d} \tilde{t}$ is constant on the scale $T$, we have by integrating (5.4) over the time for $\eta$ to change from 1 to $\eta_{\mathrm{m}}$, and using (4.2),

$$
\begin{aligned}
\frac{\mathrm{d} E}{\mathrm{~d} \tilde{t}} & =-\frac{\frac{3}{16} \int_{1}^{\eta_{\mathrm{m}}}\left(\frac{1}{\eta^{3}}-\frac{1}{\eta_{\mathrm{m}}^{3}}\right)\left(\frac{\eta^{3}}{1-\eta^{3} / \eta_{\mathrm{m}}^{3}}\right)^{\frac{1}{2}} \mathrm{~d} \eta}{\int_{1}^{\eta_{\mathrm{m}}} \frac{\eta^{\frac{3}{2}}}{\left(1-\eta^{3} / \eta_{\mathrm{m}}^{3}\right)^{\frac{1}{2}}} \mathrm{~d} \eta} \\
& =-\frac{3}{16} \eta_{\mathrm{m}}^{-3} \frac{\left\{2 \eta_{\mathrm{m}}^{\frac{1}{2}}\left(1-\eta_{\mathrm{m}}^{-3}\right)^{\frac{1}{2}}-3 \int_{\eta_{\mathrm{m}}^{-1}}^{1}\left(\frac{x^{3}}{1-x^{3}}\right)^{\frac{1}{2}} \mathrm{~d} x\right\}}{\int_{\eta_{\mathrm{m}}^{-1}}^{1}\left(\frac{x^{3}}{1-x^{3}}\right)^{\frac{1}{2}} \mathrm{~d} x} .
\end{aligned}
$$

The integral in this expression is described with sufficient accuracy by

$$
\int_{\eta_{\mathrm{m}}^{-1}}^{1}\left(\frac{x^{3}}{1-x^{3}}\right)^{\frac{1}{2}} \mathrm{~d} x \sim \frac{2}{3}\left(1-\eta_{\mathrm{m}}^{-3}\right)^{\frac{1}{2}}
$$

Then we obtain

$$
\frac{\mathrm{d} E}{\mathrm{~d} \tilde{t}}=-\frac{9}{16}\left(\eta_{\mathrm{m}}^{\frac{1}{2}}-1\right) \eta_{\mathrm{m}}^{-3}
$$

Using the expression (5.3) for $E$ and taking $\eta_{\mathrm{m}, 0}$ as the value of $\eta_{\mathrm{m}}$ at $\tilde{t}=\tilde{t}_{0}$, we find from integration of (5.6)

$$
\frac{1-\eta_{\mathrm{m}}^{-\frac{1}{2}}}{1-\eta_{\mathrm{m}, 0}^{-\frac{1}{2}}}=\exp \left\{-\left(\tilde{t}-\tilde{i}_{0}\right)\right\} .
$$

This shows that eventually $\eta_{\mathrm{m}}$ tends to 1 , or $R_{\mathrm{m}}$ to $a$. The physical explanation for this is that, since the only external force on the system is buoyancy, which is a conservative force, there is no energy input in the relative motion, as described here, and eventually the motion of the two bubbles in a pair subsides.

This takes a time, $\tilde{t}=3$, say, or $\sigma^{-1} T=3$. Therefore, while at large $\sigma$ there is no appreciable change of $\eta_{\mathrm{m}}$ in a few oscillations, $\eta_{\mathrm{m}}$ becomes unity after a time which is a multiple of $\tau$, the relaxation time. This is an interesting result and not dependent on our assumption that two bubbles in a pair have their line of centres at right angles to the direction of gravity. For, as we have seen in discussing relative motion starting at an arbitrary value of this angle, sooner or later an encounter takes place.

To what extent is this clustering of pairs observed in practice? One single pair rising in a stagnant liquid shows this behaviour (Kok 1989). Further, it is known among twophase flow researchers (Saiz-Jabardo \& Bouré 1989) that bubble clusters form in rising bubbly suspensions at the onset of transition to slug flow. However at volume concentrations well below this transition (which occurs at about $30 \%$ ) clustering together of bubbles is not observed. The reason for this could be sought in multiple collisions. 
(a)<smiles>C1CCCCC1</smiles>

\section{$\downarrow$}
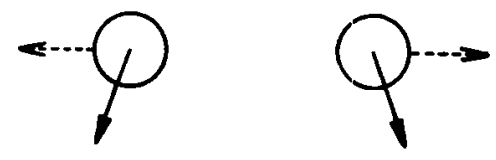

(b)
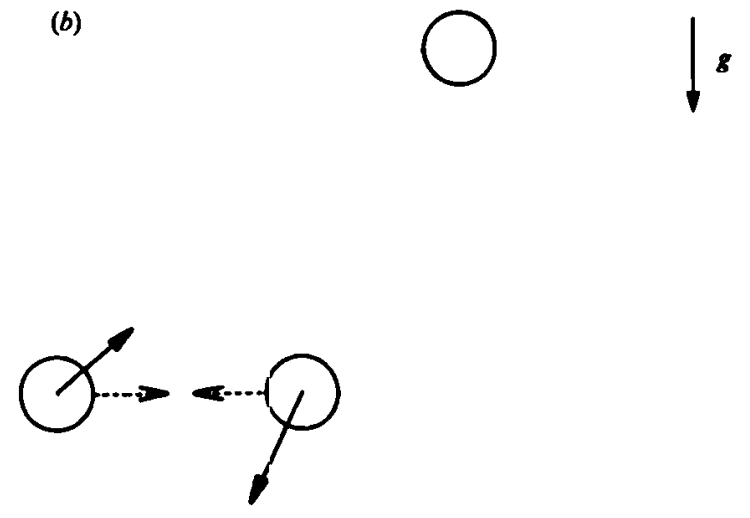

(c)
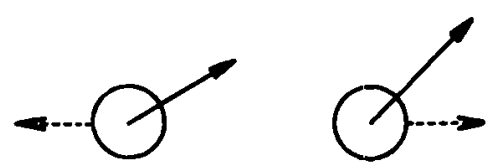

FIGURE 6. Various ways in which interaction with a third, distant, bubble increases the energy in the relative motion. The full arrows indicate direction and magnitude of the force exerted by the distant bubble, while the broken arrows indicate the direction of the relative motion of the pair.

In figure 6 some situations are sketched in which a pair is in interaction with a third bubble at some distance. In all the cases shown there, the interaction with the third bubble feeds energy in the relative motion. The question is whether such encounters of a pair with an isolated bubble are frequent enough, and the associated energy transfer 
large enough to compensate for the loss of energy due to viscous forces. We shall return to this in $\$ 9$ after the analysis of the vertical motion of bubble pairs.

If the liquid is not stagnant, but flows at such a velocity that its motion is turbulent, this turbulence will no doubt affect the motion of bubbles which are suspended in it, and vice versa, as for example shown in the experimental work of Lance \& Bataille (1991). In this paper we shall not investigate the mutual interaction between bubbles and turbulence, but restrict ourselves to phenomena with (viscous) potential flow around the bubbles.

Since this work was submitted, a numerical simulation of bubbly flow has been made by Sangani \& Didwania (1993) while a numerical calculation for a mixture in a box with periodic boundary conditions has been made by Smereka (1993). The clustering in horizontal aggregates is also observed in these works.

\section{Probability density distribution}

After the necessary preliminaries we proceed now to the calculation of the probability $P\left(x, R, R_{\mathrm{m}}, t\right)$. This is defined here such that the probability of finding one bubble in $x$ and another in $x+2 R$ at time $t$, the pair having during relative motion a maximum separation $2 \boldsymbol{R}_{\mathrm{m}}$, is given by

$$
P \mathrm{~d}^{3} x \mathrm{~d}^{3} R \mathrm{~d}^{3} R_{\mathrm{m}} .
$$

As an alternative to $\boldsymbol{R}_{\mathrm{m}}$, we could have chosen $\dot{\boldsymbol{R}}$, connected with $\boldsymbol{R}_{\mathrm{m}}$ by (5.2) (when written in physical coordinates).

Suppose there are $N$ bubbles in a volume $\mathscr{V}$, giving for the number density $\tilde{n}=$ $N / \mathscr{V}$. The probability of finding a bubble centre between $x$ and $x+\mathrm{d} x$ is $P(x) \mathrm{d}^{3} x$. The number density equals $P(x)$, since

$$
\int P(x) \mathrm{d}^{3} x=N
$$

We shall consider spatially homogeneous mixtures in which $\tilde{n}$ does not depend on $\boldsymbol{x}$. This is the case, for example, in a suspension rising under buoyancy, if we neglect the influence of the hydrostatic pressure distribution on the size of the bubbles. We therefore omit $\boldsymbol{x}$ in the argument of $\boldsymbol{P}$. Integration of $\boldsymbol{P}$ over $\boldsymbol{R}$ for fixed $\boldsymbol{x}$ must give $N$, since each bubble takes part in $N-1(=N$ for large $N)$ pairs. In our case, to get $N$ requires an additional integration over $\boldsymbol{R}_{\mathrm{m}}$, since we have divided pairs into subsets with the same energy, represented by $R_{\mathrm{m}}$. Therefore we have

$$
\iint P \mathrm{~d}^{3} R \mathrm{~d}^{3} R_{\mathrm{m}}=N \text {. }
$$

The probability density function $P$ obeys a Liouville equation

$$
\frac{\partial P}{\partial t}+\nabla_{R} \cdot(P \dot{R})+\nabla_{R_{m}} \cdot\left(P \dot{R}_{\mathrm{m}}\right)=0,
$$

which expresses the conservation of bubbles in phase space. On the short timescale for which $a / U_{\infty}$ is representative, $\boldsymbol{R}_{\mathrm{m}}$ is constant as the analysis of the preceding sections has shown. So, on this timescale there is an equilibrium distribution for given $\boldsymbol{R}_{\mathrm{m}}$. On a longer timescale $\boldsymbol{R}_{\mathrm{m}}$ changes and therefore $P$ also changes. It is therefore helpful to introduce the conditional probability density $P_{0}\left(R / R_{\mathrm{m}}\right)$ as

$$
P\left(t, R, R_{\mathrm{m}}\right)=P_{0}\left(R / R_{\mathrm{m}}\right) P\left(t, R_{\mathrm{m}}\right) .
$$


The conditional probability density expresses the probability of finding a pair with distance between them $2 \boldsymbol{R}$, given the maximum separation to be between $2 \boldsymbol{R}_{\mathrm{m}}$ and $2\left(\boldsymbol{R}_{\mathrm{m}}+\mathrm{d} \boldsymbol{R}_{\mathrm{m}}\right)$.

Moreover we make use of the analysis in $\$ 2$ where it was argued that among all possible orientations, those with the separation vector positioned in a plane with normal parallel to the direction of gravity have much higher probability than others. We take $R$ and $R_{\mathrm{m}}$ in such a plane, and write the probability of finding a pair with distance between $2 R$ and $2(R+\mathrm{d} R)$, the maximum separation being between $2 R_{\mathrm{m}}$ and $2\left(R_{\mathrm{m}}+\mathrm{d} R_{\mathrm{m}}\right)$, taking into account $(6.3)$ as

$$
4 \pi^{2} P_{0}\left(R / R_{\mathrm{m}}\right) P\left(t, R_{\mathrm{m}}\right) R R_{\mathrm{m}} \mathrm{d} R \mathrm{~d} R_{\mathrm{m}} .
$$

The interval in which $R$ can lie is between $R=a$ and $R=R_{\mathrm{m}}$. The maximum separation $2 R_{\mathrm{m}}$ is restricted to $2 a$ on one side and to some large value $2 \bar{R}$ on the other side. We shall eventually allow $\bar{R}$ to become infinite. On the short timescale the relative motion is not retarded by viscous forces. For each pair $R_{\mathrm{m}}$ is constant and in equilibrium $P$ does not depend on $t$. Taking this into account, introduction of (6.3) in (6.2) gives for $P_{0}$

$$
\frac{\mathrm{d}}{\mathrm{d} R}\left(R \dot{R} P_{0}\right)=0 .
$$

Note that, since interactions take place in the horizontal plane, $R$ is replaced by its modulus $R$. In $\S 4$ we have a solution, (4.3), for $\dot{R}^{2}$ rather than $\dot{R}$. In order to use this in solving (6.5) we multiply (6.5) with $\dot{R}$ to obtain

$$
P_{0} \dot{R}^{2}+R \dot{R}^{2} \frac{\mathrm{d} P_{0}}{\mathrm{~d} R}+\frac{1}{2} P_{0} R \frac{\mathrm{d} \dot{R}^{2}}{\mathrm{~d} R}=0 .
$$

Solving for $P_{0}$ gives, with $A\left(R_{\mathrm{m}}\right)$ being an unknown function of $R_{\mathrm{m}}$,

$$
P_{0}\left(R / R_{\mathrm{m}}\right)=A\left(R_{\mathrm{m}}\right)\left(\frac{R}{1-R^{3} / R_{\mathrm{m}}^{3}}\right)^{\frac{1}{2}} .
$$

In specifying $A\left(R_{\mathrm{m}}\right)$ we must keep in mind that $P$, decomposed according to (6.3), must satisfy (6.1). We shall require here that

$$
2 \pi \int_{a}^{R_{\mathrm{m}}} P_{0}\left(R / R_{\mathrm{m}}\right) R \mathrm{~d} R=N .
$$

Inserting (6.7) in (6.6) gives

$$
A\left(R_{\mathrm{m}}\right)=N\left\{\int_{a}^{R_{\mathrm{m}}} \frac{2 \pi R^{\frac{3}{2}} \mathrm{~d} R}{\left(1-R^{3} / R_{\mathrm{m}}^{3}\right)^{\frac{1}{2}}}\right\}^{-1} .
$$

Thus we have for $P_{0}$

$$
P_{0}\left(R / R_{\mathrm{m}}\right)=\frac{N\left\{R /\left(1-R^{3} / R_{\mathrm{m}}^{3}\right)\right\}^{\frac{1}{2}}}{\int_{a}^{R_{\mathrm{m}}} \frac{2 \pi R^{\frac{3}{2}} \mathrm{~d} R}{\left(1-R^{3} / R_{\mathrm{m}}^{3}\right)^{\frac{1}{2}}}} .
$$

From (6.1), (6.3) and (6.9) it follows that this choice for $A\left(R_{\mathrm{m}}\right)$ puts on $P\left(t, R_{\mathrm{m}}\right)$ the constraint

$$
2 \pi \int_{a}^{\bar{R}} P\left(t, R_{\mathrm{m}}\right) R_{\mathrm{m}} \mathrm{d} R_{\mathrm{m}}=1
$$


The result (6.9) can be interpreted in the following way: using (4.3) and (6.10) we see that the probability $2 \pi R P_{0}\left(R / R_{\mathrm{m}}\right) \mathrm{d} R$, of finding at given $R_{\mathrm{m}}$ the separation of a pair to be between $2 R$ and $2(R+\mathrm{d} R)$, is

$$
2 \pi R P_{0}\left(R / R_{\mathrm{m}}\right) \mathrm{d} R=N \frac{\mathrm{d} R /|\dot{R}|}{\int_{a}^{R_{\mathrm{m}}} \mathrm{d} R /|\dot{R}|} .
$$

This is apparently equal to $N$ times the time spent between $R$ and $\mathrm{d} R$, as a fraction of the total time it takes from $R=a$ to $R=R_{\mathrm{m}}$. The result (6.9) therefore looks reasonable. Information about $P\left(t, R_{\mathrm{m}}\right)$ is obtained from the Liouville equation (6.2) by integration over $R$, which gives

$$
\frac{\partial P\left(t, R_{\mathrm{m}}\right)}{\partial t}+\frac{1}{R_{\mathrm{m}}} \frac{\partial}{\partial R_{\mathrm{m}}}\left\{P\left(t, R_{\mathrm{m}}\right) \dot{R}_{\mathrm{m}} R_{\mathrm{m}}\right\}=0 .
$$

When only interaction in pairs occurs, no other processes being allowed for, no stationary probability density is possible. In fact, we can calculate the evolution in time by inserting $\mathrm{d} R_{\mathrm{m}} / \mathrm{d} \tilde{t}$, from (5.3) and (5.6), into (6.12). From (5.3) and (5.6) we have, in terms of $i=t / \tau$ and remembering that $\eta_{\mathrm{m}}=R_{\mathrm{m}} / a$,

$$
\mathrm{d} R_{\mathrm{m}} / \mathrm{d} \tilde{t}=2\left\{R_{\mathrm{m}}-a\left(R_{\mathrm{m}} / a\right)^{\frac{3}{3}}\right\} .
$$

The integrated form of this relation is (5.7) which gives, for a value $R_{\mathrm{m}}\left(\tilde{t}_{0}\right)$ at time $\tilde{t}_{0}$, a reduction at a time $\tilde{t}>\tilde{t}_{0}$ to

$$
R_{\mathrm{m}}^{\prime}=a\left[1-\left[1-\left\{a / R_{\mathrm{m}}\left(\tilde{t}_{0}\right)\right\}^{\frac{1}{2}}\right] \exp -\left(\tilde{t}-\tilde{t}_{0}\right)\right]^{-2} .
$$

The probability density $P\left(\tilde{t}, R_{\mathrm{m}}^{\prime}\right)$ for this time $\tilde{t}$ and the value $R_{\mathrm{m}}^{\prime}$ can be found from (6.12) as follows. Multiply (6.12) with $R_{\mathrm{m}}$, write $Y=R_{\mathrm{m}} P_{\mathrm{m}}$, to obtain, using (6.13) for $\dot{R}_{\mathrm{m}}$,

$$
\frac{\partial Y}{\partial t}+2\left\{R_{\mathrm{m}}-a\left(\frac{R_{\mathrm{m}}}{a}\right)^{\frac{3}{3}}\right\} \frac{\partial Y}{\partial R_{\mathrm{m}}}-\left(\frac{R_{\mathrm{m}}}{a}\right)^{\frac{1}{2}} Y=0 .
$$

The characteristics of this equation are given by (6.13) and along these we have, using (6.14)

$$
\frac{\mathrm{d} Y}{\mathrm{~d} t}=Y\left[1-\left\{1-\left(a / R_{\mathrm{m}}\left(\tilde{t}_{0}\right)\right)^{\frac{1}{2}}\right\}-\exp \left(\tilde{t}-\tilde{t}_{0}\right)\right]^{-1} .
$$

Integration of this equation, writing $R_{\mathrm{m}}^{\prime}$ instead of $R_{\mathrm{m}}$ and requiring that at $\tilde{t}=\tilde{t}_{0}$, where $R_{\mathrm{m}}=R_{\mathrm{m}}\left(\tilde{t}_{0}\right), P$ has the value $P\left(\tilde{t}_{0}, R_{\mathrm{m}}\left(\tilde{t}_{0}\right)\right)$ gives, after substituting back $P R_{\mathrm{m}}$ for $Y$,

$$
P\left(\tilde{t}, R_{\mathrm{m}}^{\prime}\right)=P\left(\tilde{t}_{0}, R_{\mathrm{m}}\left(\tilde{t}_{0}\right)\right)\left(\frac{R_{\mathrm{m}}\left(\tilde{t}_{0}\right)}{R_{\mathrm{m}}^{\prime}}\right)^{\frac{3}{2}} \exp \left(\tilde{t}-\tilde{t}_{0}\right)
$$

While (6.14) shows how in the course of time a particular value of $R_{\mathrm{m}}$ decreases, (6.15) shows that the probability density for a certain $R_{\mathrm{m}}$ increases in time. This is caused by the fact that there is in phase space an inflow, through a surface of constant $R_{\mathrm{m}}$, from larger values of $R_{\mathrm{m}}$. After a sufficiently long time this leads to a catastrophe in $R_{\mathrm{m}}=a$, or in physical terms, all pairs come together ultimately.

This is not observed in practice, at least not with a stable rising suspension. Mechanisms to prevent such catastrophes are, as discussed in $\$ 5$, multiple interactions and, at significant liquid velocity, turbulence. It should at this point be emphasized that 


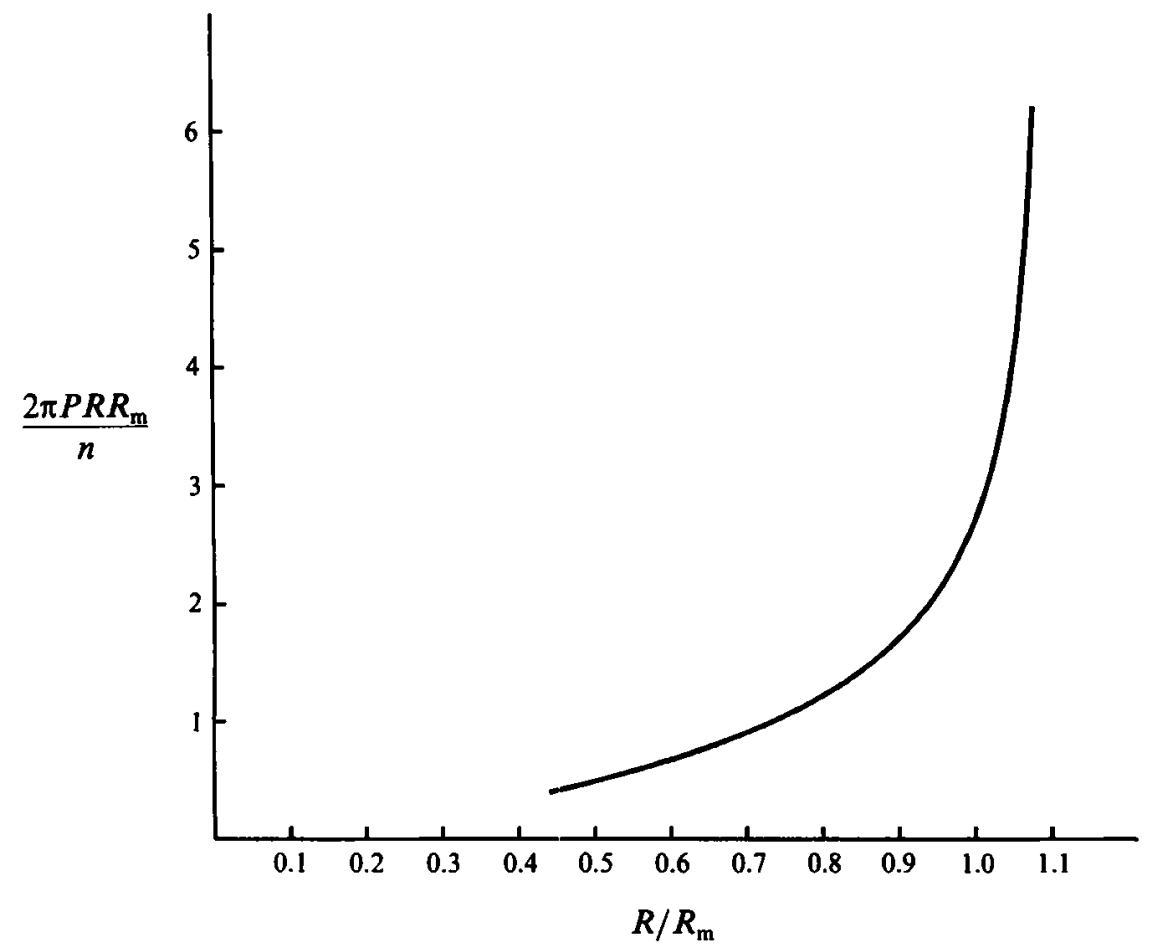

FIGURE 7. The probability density as given in (6.17), as a function of $R / R_{\mathrm{m}}$.

the conclusion of all pairs converging towards $R_{\mathrm{m}}=a$ at large times is not a result of our neglect of separation vectors oriented other than normal to the acceleration of gravity. In $\$ 4$ it has been shown that in all pairs, whatever their starting position, the bubbles ultimately approach each other along that direction. Our assumption has only been that pairs are always oriented with the separation vector in a plane with normal parallel to gravity.

In $\S 9$ we shall briefly discuss the influence of encounters of a pair with a third bubble on the behaviour of $R_{\mathrm{m}}$. For the remainder of our analysis we shall assume that $P\left(R_{\mathrm{m}}\right)$ has the same value for all $R_{\mathrm{m}}$ between $a$ and $\bar{R}$. Then it follows from (6.10) that

$$
P\left(R_{\mathrm{m}}\right)=(2 \pi)^{-1}\left\{\int_{a}^{\bar{R}} R_{\mathrm{m}} \mathrm{d} R_{\mathrm{m}}\right\}^{-1} \text {. }
$$

Taking (6.3), (6.9) and (6.16) together gives finally, with

$$
\begin{gathered}
n=N / 2 \pi \int_{a}^{\bar{R}} R_{\mathrm{m}} \mathrm{d} R_{\mathrm{m}}, \\
P\left(R, R_{\mathrm{m}}\right)=n \frac{R^{\frac{1}{2}} /\left(1-R^{3} / R_{\mathrm{m}}^{3}\right)^{\frac{1}{2}}}{\int_{a}^{R_{\mathrm{m}}} \frac{2 \pi R^{\frac{3}{2}} \mathrm{~d} R}{\left(1-R^{3} / R_{\mathrm{m}}^{3}\right)^{\frac{1}{2}}} .}
\end{gathered}
$$

In figure $7,2 \pi R R_{\mathrm{m}} P\left(R, R_{\mathrm{m}}\right) / n$ is shown for $R_{\mathrm{m}} / a=2.5$. We see that for given $R_{\mathrm{m}}, P$ increases as a function of $R$. However, integration over $R_{\mathrm{m}}$ from $R$ to $\bar{R}$ shows that the total probability of finding a separation $2 R$ decreases with $R$, because with increasing $R$ there are fewer available values of $R_{\mathrm{m}}$, namely those larger than $R$. Since the interactions take place in a plane normal to the direction of gravity the quantity $n$ is 
the number density in a plane. This can be related to the concentration $\alpha$ in the following way. Each bubble of radius $a$ cuts on the average $\frac{2}{3} \pi a^{2}$ out of a plane. The volume concentration $\alpha$ is also the total fraction of a given plane surface occupied by gas, whence

or

$$
\begin{gathered}
\alpha=\frac{2}{3} n \pi a^{2}, \\
n a^{2}=3 \alpha / 2 \pi .
\end{gathered}
$$

We conclude that if pairs of bubbles periodically approach and bounce along a horizontal line, the probability density has the form expressed in (6.17). In the next section we calculate the mean rise velocity of a mixture, based on this probability density.

\section{The mean vertical gas flux}

As discussed in $\$ 2$, the equation for relative motion in a pair is obtained by subtracting from each other the equations of the individual bubbles. Likewise, by addition we obtain an equation for the velocity of the centre of mass of the pair, because the forces exerted by one on the other cancel. This equation can, of course, be formulated with respect to many frames of reference, for example a laboratory frame, a frame moving with the liquid or a frame moving with the total volume flow. The latter has been proven to be useful in the theory of dispersions and we shall employ such a frame here. The volume flux will be indicated by $U_{0}$. The forces occurring in the force balance are

(i) a buoyancy force, $\rho g V$, where $V$ is the volume of each bubble in the pair,

(ii) a frictional force

and

$$
f(R)\left(u-U_{0}\right)
$$

(iii) the inertial reactive force which bubbles experience when their impulse changes. The impulse consists of the product of the velocity with respect to the volume-flux velocity $\left(u-U_{0}\right)$ with the virtual mass $M(R)$. This force balance has also been discussed in a recent paper by van Wijngaarden \& Kapteyn (1990), where the dependence of $M$ and $f$ on the deformation of the bubbles was also pointed out. We shall first assume bubbles to be spherical and come back later to the influence of deformations.

Taking these three forces together, gives the equation

$$
\frac{\mathrm{d}}{\mathrm{d} t}\left\{M(R)\left(u-U_{0}\right)\right\}+f(R)\left(u-U_{0}\right)=\rho g V .
$$

The functions $M(R)$ and $f(R)$ are, in view of the accuracy used here, needed only up to and including terms of order $(a / R)^{3}$. In principle they can be calculated to any order of accuracy. Referring to van Wijngaarden \& Kapteyn (1990) we have to this order

$$
\begin{aligned}
M(R) & =\frac{2}{3} \rho \pi a^{3}\left\{1+0.19(a / R)^{3}\right\}, \\
f(R) & =12 \pi \mu a\left\{1+0.13(a / R)^{3}\right\} .
\end{aligned}
$$

When we substitute (7.3) and (7.4) in (7.2), the quantities $U_{\infty}$ and $\tau$, defined in (2.2) and (2.4) respectively appear naturally and we obtain

$$
\tau \frac{\mathrm{d}}{\mathrm{d} t}\left[\left\{u-U_{0}\right\}\left\{1+0.19(a / R)^{3}\right\}\right]+\left\{u-U_{0}\right\}\left\{1+0.13\left(a / R^{3}\right)\right\}=U_{\infty} .
$$

From this we can now derive an expression for the average of $\left(u-U_{0}\right)$. However, this has to be done with some care, since (7.5) is for pairs, whereas we need an ensemble 
average over all possible realizations of $N$ bubbles. With a probability density distribution which is stationary in time, an ensemble average is the same as a time average. Averaging the terms of (7.5) we see that, with the time-independent probability function (6.17), the first term on the left-hand side of (7.5) gives no contribution. We shall focus therefore on the second term on the left-hand side of (7.5).

Note that $\left(u-U_{0}\right)$ in (7.5) is the vertical velocity of the centre of mass of a pair. Averaging of this does in general not give the average vertical bubble velocity because of vertical motion of individual bubbles with respect to the centre of mass. However, in this case relative motion of bubbles in a pair is in a horizontal plane only.

If we consider a quantity $G$, the average value of $G$ is formally

$$
\langle G(x)\rangle=\frac{1}{N !} \int G\left(x, C_{n}\right) P\left(C_{n}\right) \mathrm{d} C_{n},
$$

where $C_{n}$ denotes a configuration of $N$ bubbles. $P\left(C_{n}\right) \mathrm{d} C_{n}$ is the probability of such a configuration:

$$
P\left(C_{n}\right) \mathrm{d} C_{n}=P\left(x_{1}, x_{2}, \ldots, x_{n}\right) \mathrm{d}^{3} x_{1} \ldots \mathrm{d}^{3} x_{2} .
$$

It is the probability of finding the first of $N$ bubbles in $x_{1}$, the second in $x_{2}$, and so on. It is well known (see e.g. Batchelor (1972) that at low concentration $\alpha$,

$$
\tilde{n}\langle G\rangle=\int G(x, x+2 R) P(x, x+2 R) \mathrm{d}^{3}(2 R)+O\left(\alpha^{2}\right),
$$

where we recall that the spatial number density is indicated by $\tilde{n}$ to distinguish it from the 'number density in the plane' in, e.g. (6.18).

This reduction to an interaction between the test bubble in $x$ with only one other bubble, in $x+2 R$, is only permitted when the quantity $G$ falls off fast enough at increasing distance $2 R$. The quantity $\left(u-U_{0}\right)$ in (7.5) does not possess this property since when $R$ is large $\left(u-U_{0}\right)$ tends to $\left(U_{\infty}-U_{0}\right)$. We write therefore

$$
\left(u-U_{0}\right)=U_{\infty}+\left\{\left(u-U_{0}\right)-U_{\infty}\right\} \text {. }
$$

We introduce this into the left-hand side of (7.5) and obtain, using (7.4) and the definition (2.2) of $U_{\infty}$,

$$
\left\langle u-U_{0}\right\rangle=U_{\infty}-\left\langle 0.13 a^{3} / R^{3}\right\rangle U_{\infty} .
$$

To obtain this result, it must be kept in mind that the first term on the left-hand side of (7.5) does not contribute to the average of $\left(u-U_{0}\right)$. Further it should be recalled, that in the relation for $f(R)$ only the term in $\left(a^{3} / R^{3}\right)$ is taken into account. This is not strictly necessary since with some effort any number of terms could be included. However, in order to obtain the average indicated in (7.7), we are going to use the probability density (6.17), and this, as the reader might remember, is restricted to terms of order $\left(a^{4} / R^{4}\right)$, whereas the next terms on the right-hand side of (7.4), and also of (7.3), are of the form $(a / R)^{6}$.

We want to calculate the average $\left\langle u-U_{0}\right\rangle$, using the probability density derived in §6. As is shown by the normalization relation (6.1), the probability density $P$ there is understood as the probability of finding a bubble in $x+2 R$ given that there is one in $x$. The probability density used in (7.6) is the pair probability density. The pair probability function as used in (7.6) is obtained from (6.17) by multiplication with $n$. Bearing this in mind, evaluation of (7.7) with help of (6.17) gives

$$
\left\langle\left(u-U_{0}\right)\right\rangle=U_{\infty}-n U_{\infty} \int_{a}^{\bar{R}} 2 \pi R_{\mathrm{m}} H_{1}^{-1}\left(R_{\mathrm{m}}\right) H_{2}\left(R_{\mathrm{m}}\right) \mathrm{d} R_{\mathrm{m}},
$$


where

$$
\begin{gathered}
H_{1}\left(R_{\mathrm{m}}\right)=\int_{a}^{R_{\mathrm{m}}} \frac{R^{\frac{3}{2}} \mathrm{~d} R}{\left(1-R^{3} / R_{\mathrm{m}}^{3}\right)^{\frac{1}{2}}}, \\
H_{2}\left(R_{\mathrm{m}}\right)=0.13 a^{3} \int_{a}^{R_{\mathrm{m}}} \frac{\mathrm{d} R}{R^{\frac{3}{2}}\left(1-R^{3} / R_{\mathrm{m}}^{3}\right)^{\frac{1}{2}}} .
\end{gathered}
$$

The integral in (7.9) is of the form given in (5.5) and hence is

$$
H_{1}\left(R_{\mathrm{m}}\right) \approx \frac{2}{3} R_{\mathrm{m}}^{\frac{5}{2}}\left(1-a^{3} / R_{\mathrm{m}}^{3}\right)^{\frac{1}{2}}
$$

$H_{2}$ can be reduced to an integral of the form of $H_{1}$ by partial integration, which gives

$$
H_{2}=0.13 a^{\frac{5}{2}}\left[2\left(1-a^{3} / R_{\mathrm{m}}^{3}\right)^{\frac{1}{2}}-\frac{4}{3}\left(a / R_{\mathrm{m}}\right)^{3}\left(R_{\mathrm{m}} / a\right)^{\frac{5}{2}}\left(1-a^{3} / R_{\mathrm{m}}^{3}\right)^{\frac{1}{2}}\right] .
$$

Introducing these results for $H_{1}$ and $H_{2}$ in (7.8) gives

$$
\begin{aligned}
\left\langle u-U_{0}\right\rangle & =U_{\infty}\left\{1-0.13 \pi n a^{\frac{5}{2}}\left(3 \int_{a}^{\bar{R}} R_{\mathrm{m}}^{-\frac{3}{2}} \mathrm{~d} R_{\mathrm{m}}-2 a^{\frac{1}{2}} \int_{a}^{\bar{R}} R_{\mathrm{m}}^{-2} \mathrm{~d} R_{\mathrm{m}}\right)\right\} \\
& =U_{\infty}\left\{1-1.04 \pi n a^{2}\right\},
\end{aligned}
$$

with $\bar{R} \rightarrow \infty$. Finally, by using (6.18), this gives in terms of the volume concentration $\alpha$ the result

$$
\left\langle u-U_{0}\right\rangle=U_{\infty}\{1-1.56 \alpha\}+O\left(\alpha^{2}\right) .
$$

Using the complete series for $f(R)$ in (7.4) gives 1.43 instead of 1.56 in (7.11). However, it is hard to say whether this is more accurate since, as we have stressed, terms of order $(a / R)^{4}$ and higher have been neglected in calculating the probability density.

\section{Comparison with experiments, and discussion}

The result (7.11) is valid under the assumption, made in the theory, of spherical bubbles, all of the same size. The only experiments reported in the literature where the latter condition is satisfied are those by van Wijngaarden \& Kapteyn (1990). They carried out experiments with suspensions consisting of filtrated tap water and bubbles with an effective radius of $1.4 \mathrm{~mm}$, with a spread of $0.25 \mathrm{~mm}$. These bubbles are certainly not spherical, but oblate spheroids with a ratio of longer axis to shorter axis of about 1.8 (van Wijngaarden \& Kapteyn 1990). The measured value of $U_{\infty}$ for these bubbles is

$$
U_{\infty}=0.27 \mathrm{~m} / \mathrm{s},
$$

which is very close to the value given in Clift, Grace \& Weber (1978) for pure water. The speed of rising of uniform suspensions of these bubbles in water was measured by van Wijngaarden \& Kapteyn (1990), with the result

$$
\left\langle u-U_{0}\right\rangle=0.223(1-1,78 \alpha), \quad 0.02<\alpha<0.15 \text {. }
$$

It is interesting to note that the constant outside the brackets in (8.2) is smaller than $U_{\infty}$. Of course, for $\alpha \Rightarrow 0, u-U_{0}$ must approach $U_{\infty}$. The linear behaviour in (8.2) leads apparently to a lower value, when extrapolated to $\alpha=0$, which indicates that between $\alpha=0$ and $\alpha=0.02\left(u-U_{0}\right)$ changes more rapidly than linearly. The slope $\mathrm{d}\left\langle u-U_{0}\right\rangle / \mathrm{d} \alpha$ is according to these measurements constant between $\alpha=0.02$ and 0.15 and has the value, from (8.2), of 0.40 . The corresponding value of this slope predicted by our theory is, from (7.11) and using (8.1), 0.42 which is close enough.

In the context of his experiments on void fraction waves Bouré measured the speed of rising of uniform bubbly suspensions. In these experiments the spread of bubble 
sizes is larger (Bouré, private communication) then in the experiments by van Wijngaarden \& Kapteyn (1990). Bouré (1988) gives the result

$$
\left\langle u-U_{0}\right\rangle=0.22(1-2.25 \alpha) \text {. }
$$

The coefficient preceding $\alpha$ is somewhat larger than in (8.2) but this can be attributed to bubble deformation.

In our analysis we have considered only spherical bubbles. In practice, the bubbles under discussion for which the Reynolds number of the relative motion is large will be deformed. Deviations from the spherical shape affect properties like virtual mass and frictional force and therefore will also have an effect on the average velocity of rising. Because oblate spheroidal bubbles have a larger resistance than spherical bubbles of the same volume, one would expect that, other things being equal, the speed of rising of a suspension of oblate spheroidal bubbles is somewhat lower than with spherical bubbles. It should be interesting to investigate the influence of deformation on the analysis in this paper, although it will be difficult to account for fluctuations in the shape during interaction. Including the influence on the average shape is feasible as shown in van Wijngaarden (1991), where the effect of deformation on the stability of voidage waves in bubbly flows is investigated.

In the writer's opinion the present work shows, based on physical arguments, that for bubbles of the size of a few millimetres, characteristic times are such that interaction can be analysed using potential flow theory for the relative motion. The result for the probability density hinges, apart from the description of the flow as potential flow, on the assumptions of bouncing and of interactions taking place mainly in a plane. The bouncing of an isolated pair of bubbles is clearly shown in the work of Kok (1989) reported in $\S 3$. Regarding the second assumption, the present author feels that the analysis together with the numerical results by Kok (1989) warrants this assumption.

Finally, in order to get some more insight in the meaning of our result, we look at what other probability distributions give as mean velocity of rise. First we take randomly distributed bubbles. Then,

$$
\left.\begin{array}{ll}
P(x \mid x+2 R)=0 & \text { when } R<a \\
P(x \mid x+2 R)=\tilde{n} & \text { when } R>a .
\end{array}\right\}
$$

The liquid velocity $v_{1 n d}$ induced in $x$ by the bubble in $x+2 R$ is

$$
v_{\text {ind }}-U_{0}=\frac{U_{\infty} a^{3}}{8 R^{3}}\left\{\frac{3(e \cdot R) R}{R^{2}}-e\right\},
$$

which is the velocity induced in $x+2 R$ by a dipole of strength $\frac{1}{2} U_{\infty} a^{3}$ in the direction $e$, situated in $x$. In the absence of this induced velocity the bubble in $x$ would attain the velocity $U_{\infty}$ with respect to $U_{0}$, in the direction of $e$. In the presence of the other bubble buoyancy must now also overcome the induced velocity expressed in (8.5). In van Wijngaarden \& Kapteyn (1990) it is shown that, with velocity $u$ of the bubble in $x$, its drag is

$$
12 \pi \mu a\left(u-U_{0}\right)\left\{1-\frac{2\left(v_{\text {ind }}-U_{0}\right) \cdot\left(u-U_{0}\right)}{\left(u-U_{0}\right)^{2}}\right\} .
$$

The average of this in the direction $e$ must equal $\rho g V$. This means that

$$
\left\langle\left(u-U_{0}\right) \cdot e\right\rangle-\left\langle 2\left\{\left(u-U_{0}\right) \cdot e\right\}\left\{\left(v_{\text {ind }}-U_{0}\right) \cdot\left(u-U_{0}\right)\right\} /\left(u-U_{0}\right)^{2}\right\rangle=U_{\infty} .
$$

In the second term $u-U_{0}$ can be replaced by $U_{\infty} e$, whence

$$
\left\langle\left(\boldsymbol{u}-U_{0}\right) \cdot e\right\rangle=U_{\infty}+\left\langle 2\left(v_{1 n d}-U_{0}\right) \cdot e\right\rangle .
$$


Carrying out the averaging using (8.4) and (8.5) we encounter the familiar phenomenon of non-uniformly convergent integrals. This difficulty can be overcome by Batchelor's (1972) renormalization method. In this case this means noting that, when $v$ denotes the local velocity vector either in gas or in liquid, we have by definition

$$
\int\left(v-U_{0}\right) P\left(C_{N}\right) \mathrm{d} C_{N}=0 .
$$

Subtracting the conditional and unconditional average, we may write in the twobubble approximation

$$
2\left\langle\left(v-U_{0}\right) \cdot e\right\rangle=2 \int\left\{\left(v-U_{0}\right\}\{P(x \mid x+2 R)-P(x)\} \mathrm{d}^{3} R .\right.
$$

From (8.4) it follows that the region $R>a$ does not contribute to the integral on the right-hand side. The region $0<R<\frac{1}{2} a$, where $v \cdot e=U_{\infty}$, contributes to the first order in $\alpha$,

$$
2 \tilde{n} \int_{\text {sphere }}-U_{\infty} \mathrm{d}^{3} R=-2 \alpha U_{\infty} .
$$

In the region $\frac{1}{2} a<R<a$, the velocity $v-U_{0}$ can be approximated by the right-hand side of (8.5) which gives a zero contribution. The result is therefore, from (8.6)-(8.8),

$$
\left\langle u-U_{0}\right\rangle=U_{\infty}(1-2 \alpha) \text {. }
$$

The coefficient in the $\alpha$-term is larger than the corresponding one (1.56) in our result (7.11), and also than in the $\alpha$-term in the experimental result (8.2). The expression on the right-hand side of (8.9) is an average over pair configurations, among which there are many with rise velocity larger than $U_{\infty}$. Nevertheless the result is smaller than that of a calculation in which all lines of centres are horizontal and pairs move slower than $U_{\infty}$. This looks surprising at first sight. The reason is in the displacement flow. In the region inaccessible to other bubble centres, $\frac{1}{2} a<R<a$, bubbles carry fluid with them, in principle. This is

$$
\int_{a / 2<R<a} v \cdot e \mathrm{~d}^{3} R \text {. }
$$

For randomly distributed bubbles, we saw above that this is zero, giving no need for a compensating fluid flow. However, if pairs are considered with only horizontal lines of centres, the above integral gives, evaluated with (8.5), $-\frac{1}{4} \pi U_{\infty} a^{2}$. This is a downflow which is compensated by a corresponding upflow $\frac{1}{4} \pi U_{\infty} n a^{2}=\frac{3}{8} U_{\infty} \alpha$, in the space accessible to bubble centres.

Therefore doing the calculation of the mean rise velocity for pairs, arranged in a horizontal plane, but with $P(R)$ constant, for $R>a$, we find

$$
\left\langle u-U_{0}\right\rangle-=U_{\infty}-2 \alpha U_{\infty}+\frac{3}{4} \alpha U_{\infty}=U_{\infty}(1-1,25 \alpha) \text {. }
$$

This is larger than the result $U_{\infty}(1-1.56 \alpha)$, found in $\S 7$ (cf. (7.11)). Both are for pairs in a horizontal plane, but in the calculation leading to $(7.11), P(R)$ decreases with increasing $R$, as mentioned in connection with (6.17). This means that there are, relative to the random distribution, less pairs with larger $R$ and correspondingly relatively high rise velocity.

\section{Interaction between a bubble pair and a distant third one}

In $\$ 6$ we found that, under the restriction of binary interactions, the kinetic energy of relative motion of a pair gets exhausted gradually, with clustering as an eventual result. It was suggested that multiple encounters inject energy in the relative motion, 


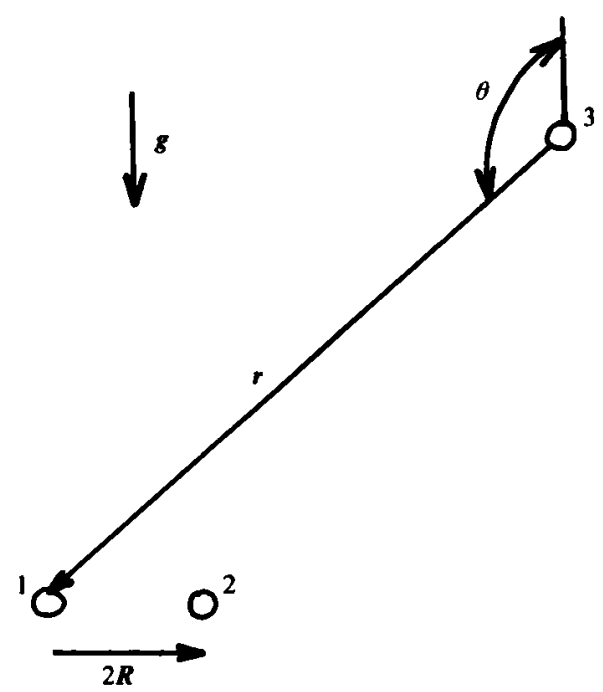

FIGURE 8 . A bubble pair 1,2 , distance $2 \boldsymbol{R}$ apart, in interaction with a distant third one, 3 , at distance $r$ from $1 . \theta$ is the angle between $r$ and the vertical direction.

thereby counteracting the tendency to clustering. The natural candidate for representing multiple interactions is the interaction between a pair and a, distant, third bubble. We consider this here, as sketched in figure 8 .

Bubble 1 , in $x$, and bubble 2, in $x+2 R$, form the pair. The third one is in $x+r$. In the foregoing sections we have, in fact, considered the situations where $r$ is so large that its influence on the pair can be neglected. Here we look at 'third' bubbles for which $r$ is still large with respect to $a$ or $R_{\mathrm{m}}$ (a multiple of order one of $a$ ), but not infinite.

During one full cycle whereby $R$ increases from $a$ to $R_{\mathrm{m}}$ and back, bubbles 1 and 2 experience a viscous force of average magnitude

$$
\begin{aligned}
& \frac{1}{t\left(R_{\mathrm{m}}\right)-t(a)} \int_{t(a)}^{t\left(R_{\mathrm{m}}\right)} 12 \pi \mu a \dot{R}\left(t^{\prime}\right) \mathrm{d} t^{\prime} \\
& \quad=\left\{\int_{a}^{R_{\mathrm{m}}}\left(\frac{\mathrm{d} t}{\mathrm{~d} R}\right) \mathrm{d} R\right\}^{-1} \int_{a}^{R_{\mathrm{m}}} 12 \pi \mu a \mathrm{~d} R \\
& \quad=8 \pi \mu a U_{\infty}\left(a / R_{\mathrm{m}}\right)^{\frac{3}{2}}\left(1-a / R_{\mathrm{m}}\right)\left(1-a^{3} / R_{\mathrm{m}}^{3}\right)^{-\frac{1}{2}} .
\end{aligned}
$$

Now we consider bubble 3 , at distance $r\left(r \gg R_{\mathrm{m}}\right)$ from 1 . The force exerted by 3 on 1 and given, with $2 R$ instead of $r$, by the right-hand side of (2.5) and (2.6), can be expressed as the gradient of a potential $\Omega$, say, given by

$$
\Omega=\frac{3}{32} \rho U_{\infty}^{2} a^{6} / r^{3}\left(3 \cos ^{2} \theta-1\right) .
$$

The special case $\theta=\frac{1}{2} \pi$ is mentioned in the beginning of $\S 5$. If the same force were exerted by 3 on 2 , no relative motion would be induced. We must look therefore at the difference of $\nabla \Omega$ evaluated at $x$ and at $x+2 R$. Moreover, we need the component of this in the direction of $\boldsymbol{R}$. Let $\boldsymbol{e}$ be a unit vector in that direction. Then, at given separation $2 R$, the resultant force in the direction $e$ is

$$
G=(-2 R \cdot \nabla \nabla \Omega) \cdot e .
$$

When, at the considered position, $\dot{R}$ is in the direction of $\boldsymbol{R}$ this force increases the energy in the relative motion. At fixed $r$ there is a second instant during a cycle at which 
the separation is again $2 \boldsymbol{R}$, whereby energy is reduced by the same amount. However, during the interval bubble 3 does not remain at the same place but moves with respect to the pair formed by 1 and 2 .

According to (7.7) bubble 3 outruns the pair over a distance of about $0.13 a^{3} / R_{\mathrm{m}}^{3} U_{\infty}$ per unit time. The exact value of this distance, which we denote with $\Delta z, z$ being a coordinate in the vertical direction, in the time interval between two positions with the same $R$, from (7.7), is

$$
\frac{\Delta z}{a}=0.26 U_{\infty} a^{2} \int_{t(R)}^{t\left(R_{\mathrm{m}}\right)} \frac{\mathrm{d} t}{R^{3}}=\frac{0.52 a^{2}}{R_{\mathrm{m}}^{2}}\left[\left\{\left(\frac{R_{\mathrm{m}}}{R}\right)^{\frac{1}{2}}-\frac{2}{3}\right\}\left\{1-\left(\frac{R^{3}}{R_{\mathrm{m}}^{3}}\right)^{\frac{1}{2}}\right\}\right] .
$$

For a given value of $R$ the net force, injecting kinetic energy in the relative motion, is the difference between $G$ at position $r$ and a position $r+\Delta z$ in the vertical direction. Since (9.4) shows that $\Delta z$ is of order $a$, and therefore small with respect to $r$, to evaluate this difference we may expand $G$ around $r$,

$$
G(\Delta z)=\Delta z\left(\frac{\partial G}{\partial z}\right)_{r}+\frac{1}{2}(\Delta z)^{3}\left(\frac{\partial^{2} G}{\partial z^{2}}\right)+\ldots
$$

To find the average of the right-hand side of $(9.5)$, we must evaluate the $z$-derivatives with help of (9.3), integrate over $\boldsymbol{r}$ and a full cycle of $\boldsymbol{R}$, after multiplication with the probability density $P(x+r)$. In the present context, which is to make an estimate of the average inertial force exerted by bubble 3 , we ignore the effect of the interaction on the probability density and take $P(x+r)=\tilde{n}$. From (9.2) and (9.3) it follows $(z=r \cos \theta)$, that $\partial G / \partial z$ is an odd function of $z$ and therefore the first term on the right-hand side of $(9.5)$ does not contribute to the integral over $r$. We calculate therefore the average of the second term on the right-hand side of (9.5).

Introducing $x_{R}$ as an orthogonal coordinate in the plane normal to $g$, in the direction of $\boldsymbol{R}$, we have to evaluate, from (9.3)-(9.5)

$$
\langle G\rangle=\frac{(0.52)^{2} a^{8} \tilde{n}}{\left(R_{\mathrm{m}}-a\right) R_{\mathrm{m}}^{4}} \int \frac{\partial^{4} \Omega}{\partial x_{R}^{2} \partial_{\mathrm{z}}^{2}} \mathrm{~d}^{3} r \int_{a}^{R_{\mathrm{m}}} 2 R\left(1-\frac{R^{3}}{R_{\mathrm{m}}^{3}}\right)\left(\frac{R_{\mathrm{m}}^{\frac{1}{2}}}{R^{\frac{1}{2}}}-\frac{2}{3}\right)^{2} \mathrm{~d} R .
$$

The integral over $R$ gives $0.58 R_{\mathrm{m}}^{2}$ as the leading term, whereupon integration of the remaining expression with use of $(9.2)$ results in, to leading order in $\left(a / R_{\mathrm{m}}\right)$,

$$
\langle G\rangle \approx 0.02 \alpha \rho U_{\infty}^{2} a^{2}\left(a^{3} / R_{\mathrm{m}}^{3}\right),
$$

where use has been made of the relation

$$
\alpha=\frac{4}{3} \pi \tilde{n} a^{3} .
$$

To the same accuracy, in terms of $a / R_{\mathrm{m}}$, the average frictional force is, from (9.1),

$$
\langle F\rangle \sim 8 \pi \mu a U_{\infty}\left(a / R_{\mathrm{m}}\right)^{\frac{3}{2}}
$$

From (9.6) and (9.7) we find for the ratio $\langle G\rangle /\langle F\rangle$,

$$
\langle G\rangle /\langle F\rangle=0.01 \alpha \sigma\left(a / R_{\mathrm{m}}\right)^{\frac{3}{2}},
$$

where the definitions (2.4) and (2.10) for $\tau$ and $\sigma$, respectively, have been used.

This could be significant for large $\sigma$ and not too small $\alpha$. When $\sigma=10^{2}, \alpha=10 \%$, $R_{\mathrm{m}}=3 a$, the right-hand side of $(9.8)$ is only 0.02 . This suggests that multiple encounters may be significant, but presumably not able to compensate for the tendency to cluster which we found in our study of binary interactions. 
During the preparation of this paper the author enjoyed very useful discussions with A. Biesheuvel and J. H. Lammers.

\section{REFERENCES}

BATCHELOR, G. K. 1972 Sedimentation in a dilute dispersion of spheres. J. Fluid Mech. 52, 245-268.

BAtCheloR, G. K. 1974 Transport properties of two-phase materials with random structure. Ann. Rev. Fluid Mech. 6, 227-255.

BIESHEUVEL, A. 1984 On void fraction waves in dilute mixtures of liquid and gas bubbles. Thesis, University of Twente.

BIEsheuvel, A. \& WiJngaARden, L. van 1982 The motion of pairs of gas bubbles in a perfect fluid. J. Engng Maths 16, 349-365.

Biesheuvel, A. \& WungaARDEN, L. VAN 1984 Two-phase flow equations for a dilute dispersion of gas bubbles in liquid. J. Fluid Mech. 148, 301-318.

BouRÉ, J. 1988 Properties of kinematic waves in two-phase flows. Presented at European Two-Phase Flow group Meeting, Brussels, May 30th-June 1st.

Chesters, A. K. 1991 The modelling of coalescence processes in fluid-liquid dispersions: A review of current understanding. Trans. Inst. Chem. Engng 69, 259-270.

Clift, R., Grace, R. J. \& Weber, M. E. 1978 Bubbles, Drops and Particles. Academic.

KAPTEIJ, C. 1989 Measurements on concentration waves in bubbly liquids. Thesis, University of Twente.

KIRKPATRICK, R. D. \& LOCKETT, M. J. 1974 The influence of approach velocity on bubble coalescence. Chem. Engng Sci. 29, 2363-2373.

KoK, J. B. W. 1989 Dynamics of gas bubbles moving through liquid. Thesis, University of Twente.

LanCE, M. \& Bataille, J. 1991 Turbulence in the liquid phase of a uniform bubbly air-water flow. J. Fluid Mech. 222, 95-119.

Levich, V. G. 1962 Physicochemical Hydrodynamics. Prentice Hall.

MOORE, D. W. 1963 The boundary layer on a spherical gas bubble. J. Fluid Mech. 16, 161-176.

MOORE, D. W. 1965 The velocity of rise of distorted gas bubbles in a liquid of small viscosity. J. Fluid Mech. 23, 749-766.

SAIz-JABARDo, J. M. \& BouRÉ, J. A. 1989 Experiments on void fraction waves. Intl J. Multiphase Flow 15, 483-493.

Sangani, A. S. \& Didwania, A. K. 1993 Dynamic simulations of flows of bubbly liquids at large Reynolds numbers. J. Fluid Mech. 250, 307-337.

SMEREKA, P. 1993 On the motion of bubbles in a periodic box. J. Fluid Mech. to appear.

WIJNGAARDEN, L. VAN 1976 Hydrodynamic interaction between gas bubbles in liquid. J. Fluid Mech. 77, $27-44$.

WiJngaARden, L. vaN 1991 Bubble deformation in bubbly liquids and its effect on the stability of voidage waves. In Mathematical Approaches in Hydrodynamics (ed. T. Miloh), pp. 38-49. SIAM.

WijngaARDEN, L. vaN. \& KAPTEIJN, C. 1990 Concentration waves in dilute bubble/liquid mixtures. J. Fluid Mech. 212, 111-137. 